\title{
Trends in Hospitalization and In-Hospital Mortality with Inflammatory Bowel Disease Complicated By Venous Thromboembolism
}

\author{
Rajkumar Doshi ${ }^{1}$, Shreyans Doshi ${ }^{2}$, Jiten Desai ${ }^{3}$, Kazumi Khamar ${ }^{4}$, John Hudson ${ }^{5}$ and Nihar \\ Shah $^{4^{*}}$ \\ ${ }^{1}$ North Shore University Hospital, Manhasset, New York 11030, USA \\ ${ }^{2}$ University of Central Florida College of Medicine/HCA Graduate Medical Education Consortium, Gainesville, Florida, \\ 32605, USA
}

${ }^{3}$ Nassau University Medical Center, East Meadow, New York, 11554, USA

${ }_{4}^{4}$ Joan C. Edward School of Medicine at Marshall University, Huntington, West Virginia, 25755, USA

${ }^{5}$ Marshall University School of Pharmacy, Huntington, West Virginia, 25755, USA

Received: June 06, 2017; Accepted: July 24, 2017; Published: August 18, 2017

"Corresponding author: Nihar Shah, Assistant Professor, Department of Internal Medicine Section of Gastroenterology, Joan C. Edward school of Medicine Marshall University, USA, Tel: +1 3046911000 ; E-mail: Shahniharn@gmail.com

\begin{abstract}
Background: Hospitalized patients with Inflammatory Bowel Disease (IBD) are at increased risk of Venous Thromboembolism (VTE), Including Deep Vein Thrombosis (DVT) and Pulmonary Embolism (PE), and these conditions are associated with increased in-hospital mortality, morbidities and longer length of stay. Our goal is to learn trends in Hospitalization and In-Hospital Outcomes with IBD Complicated by VTE.
\end{abstract}

Methods: In this cross-sectional study, hospitalization data were extracted from National Inpatient Sample (NIS) between 2005- 2014. International classification of disease, ninth revision, clinical modification codes (ICD 9 CM) were used to identify VTE and IBD. We have included all patients with primary or secondary diagnosis of VTE and IBD. Primary outcomes were inhospitalization mortality and average length of stay. Our secondary outcomes were blood loss requiring transfusion, gastrointestinal hemorrhage and postoperative wound infection. Trend analysis was performed using JonchheereTerpstra Test.

Result: A total of 16,550 (weighted 82750) patients were included in our final analysis. Overall hospitalizations for IBD complicated by VTE are rising from 2005 to 2014. Overall, in-hospital mortality was $4.7 \%$ in IBD patients complicated by VTE. In-hospital mortality in IBD patients was $0.18 \%$ in 2005 , which increased to $0.52 \%$ in 2014 ( $P$ for trend $=<0.0001$ ). Average length of stay in IBD patients was 9.6 days in 2005 which increased to 10.9 days in 2014 ( $\mathrm{P}$ for trend $=<0.0001$ ).

Conclusion: Overall hospitalizations, in-hospital mortality and average length of stay for IBD complicated by VTE are rising since last decade; which is a serious health care concern. It is also suggestive of need to perform more detailed research in this area.

Key Words: Venous Thromboembolism; Inflammatory Bowel Disease; Trends; In-Hospital Outcomes

Abbreviations: IBD- Inflammatory Bowel Disease; VTE- Venous Thromboembolism; EIM- Extra Intestinal Manifestation; DVT- Deep Venous Thrombosis; PE- Pulmonary Embolism; NIS- National Inpatient Sample; USUlcerative Colitis; CD- Crohn's Disease; CCI- Charlson's Comorbidity Index

\section{Introduction}

An Inflammatory Bowel Disease (IBD) is a chronic disorder that predominantly affects the bowel; however, it can also be associated with numerous extra intestinal complications. Among these complications, Venous Thromboembolism (VTE) is particularly important due to its significant morbidity and mortality. Although less than 10 percent of patients with Inflammatory Bowel Disease (IBD) have an Extra Intestinal Manifestation (EIM) at initial presentation, 25 percent of patients have an EIM in their lifetime [1]. The possible association between IBD and VTE was first reported in 1936 by Bargen and Barker, who described 18 patients with thromboembolic disease from among more than 1000 patients treated for IBD at the Mayo Clinic [2]. Patients with IBD are at three to four times higher risk of VTE compared to non-IBD subjects and they are also affected at a younger age [3-5]. The etiology of VTE is multifactorial; active flares, fistulas, and abscesses are present in the majority of IBD patients at the time of the thromboembolic event. Deep Venous Thrombosis (DVT) of the lower and upper extremities and pulmonary embolism (PE) are the most common locations of VTE in IBD patients, but also uncommon sites of thrombosis, including portal, mesenteric, cerebrovascular or retinal veins, have been also noted [6]. IBD patients are more commonly exposed to risk factors that may provoke VTE, including dehydration, surgery, immobilization and central venous catheters. The major complications of VTE are death and recurrence of thromboembolism.

VTE prevention involves correcting modifiable risk factors, such as disease activity, vitamin deficiency, dehydration and prolonged immobilization. Recurrent VTE can be prevented by anticoagulant treatment, which can cause fatal bleeding. Thus, choosing the optimal duration of prophylaxis for an individual patient entails balancing the risk of bleeding against the risk of recurrent VTE [7-10]. 


\section{Methods}

\section{Data Source and Study Population}

Hospitalization data were extracted from National Inpatient Sample (NIS) between 2005-2014 [11]. The NIS is a $20 \%$ stratified sample of around 1000 hospitals in the United States and is maintained as part of the Healthcare Cost and Utilization Project (HCUP) sponsored by the Agency for Healthcare Research and Quality (AHRQ) [11]. Each patient discharged from one of this hospital has age, gender, race, admission type (elective or non-elective), primary payer, median house hold income, teaching status of the hospital, hospital region, disposition, primary and secondary diagnosis, primary and secondary procedures, and length of stay. The NIS is a publically accessible dataset and contains completely deidentified data. Hence, use of this database was except from institutional review board committee approval.

We have included all patients with primary or secondary diagnosis of VTE and IBD. We have used International classification of disease, ninth revision; clinical modification codes (ICD 9 CM) to identify VTE
$(325,451.11,451.19,451.2,451.81,451.83,451.84,452,572.1,453.0-$ 453.3, 453.40-453.42, 453.8, 453.9, 415.1, 415.11, 415.12, and 415.19) and IBD (555 and 556). All patients below 18 years of age were excluded from our study. Patient's selection chart is shown in figure 1. IBD groups have all the patients included in our study. "Both" category included those patients who had Ulcerative Colitis (UC) and Crohn's Disease (CD) as their diagnosis. We used comorbidities given in the database which has been used previously [12]. For disease presentation, we have used appropriate ICD 9 CM procedural and diagnostic codes to identify active fistulizing disease or intra-abdominal abscess, active stricturing disease, bowel obstruction, perianal abscess, lower gastrointestinal bleeding, hypovolemia, and malnutrition [Supplementary Table 1] [13]. For in-hospital outcomes, we have used appropriate ICD 9 CM procedural and diagnostic codes to identify blood loss requiring transfusion, gastrointestinal hemorrhage, postoperative wound infection, and surgical complications [Supplementary Table 1] [13]. Appropriate ICD 9 CM codes were used to examine surgical complications [Supplementary Table 1] [13].

\begin{tabular}{|c|c|}
\hline \multicolumn{2}{|l|}{ Supplementary Table 1: ICD 9 CM Codes Used for Our Study } \\
\hline Variable Name & ICD 9 CM Codes \\
\hline Venous Thromboembolism & $\begin{array}{c}325,451.11,451.19,451.2,451.81,451.83,451.84,452,572.1,453.0-453.3,453.40-453.42,453.8 \\
453.9,415.1,415.11,415.12, \text { and } 415.19\end{array}$ \\
\hline Crohn's Disease & 555 \\
\hline Ulcerative Colitis & 556 \\
\hline Active Fistulizing disease or intra-abdominal abscess & $537.4,567.2,569.5,593.3,596.1,619.1,567.21,567.22,569.81,569.83$ \\
\hline Active Stricturing Disease & $560.9,537.3$ \\
\hline Bowel Obstruction & 560,568 \\
\hline Perianal Abscess & 566 \\
\hline Unspecified Lower GI Bleeding & $578.9,569.3$ \\
\hline Hypovolemia & 276.5 \\
\hline Malnutrition & 263 \\
\hline Endoscopic Procedure & $45.1,45.2,45.3,45.4,45.52,46.86$ \\
\hline Incision, Excision and Anastomosis of Intestine & $45.0,45.9,45.5,45.6,45.33,45.50,45.51,45.61,45.62,45.63,45.90,45.91,45.93,45.94$ \\
\hline Resection of Large Intestine & $45.7,45.8,45.81,45.82,45.83,45.92,45.93,45.94,45.95$ \\
\hline Rectal and perirectal Surgery & 48 \\
\hline Other Surgery of small intestine & $\begin{array}{c}46.0,46.4,46.5,46.6,46.01,46.02,46.20,46.21,46.22,46.23,46.24,46.40,46.41,46.50,46.51,46.60,46.61,4 \\
6.62,46.71,46.72,46.73,46.74,46.81,46.95\end{array}$ \\
\hline Other Surgery of large intestine & $\begin{array}{c}46.1,46.03,46.04,46.10,46.11,46.13,46.14,46.42,46.43,46.52,46.63,46.64,46.75,46.76,46.79,46.82,46.8 \\
7,46.91,46.92,46.96\end{array}$ \\
\hline $\begin{array}{l}\text { Post-operative wound complication including infection, } \\
\text { dehiscence, and fistula }\end{array}$ & $998.6,998.83,998.12,998.13,998.31,998.32$ \\
\hline
\end{tabular}

\section{Statistical Analysis}

SAS 9.4 (SAS institute, Cary, NC) was used to perform statistical analysis. Continuous variables are presented as mean \pm standard deviation. Categorical variables are expressed as frequencies in percentages. Trend analysis performed using Jonchheere-Terpstra Test. Trend charts were created in Microsoft excel 2010. In-Hospital mortality predictors were analyzed using multiple logistic regression models. Variables included in the analysis were: Age, gender, procedure type, primary payer, hospital location and Charlson's comorbidity score. Statistical significance was set at $0.05(95 \%)$.

\section{Results}

A total of 16,550 (weighted 82750) patients were included in our final analysis. Predominantly, females were (53.9\%) higher compared to males. Similarly, Whites (80.5\%) were predominant in our study. Severity 
of co-morbid conditions was defined using Deyo modification of Charlson Co-morbidity Index (CCI). 49\% population had no comorbidity and 22.9\% had at least one comorbidity as seen by CCI. Most common primary payer was Medicare (47\%) followed by private insurance (37.5\%). Highest patient population with VTE in IBD was noted from urban teaching hospital (55\%). Regional differences existed, with highest patients were seen from South America (35.7\%) [Table 1]. Highest comorbid conditions prevalent in our study population were hypertension (39.7\%), fluid and electrolyte disorder $(37.1 \%)$, deficiency anemia (31.6\%), and chronic pulmonary disease (18.5\%) [Table 2]. On presentation, $10.8 \%$ with malnutrition, $10.6 \%$ population with bowel obstruction, $9.7 \%$ with hypovolemia, $7.1 \%$ with active fistulizing disease or intraabdominal abscess, $5.2 \%$ with unspecified lower gastrointestinal bleeding, $2.6 \%$ with active stricturing disease were present [Table 3].

Table 1: Demographic Characteristics of Patients Hospitalized with Venous Thromboembolism, Stratified by IBD, UC, CD and Both

\begin{tabular}{|c|c|c|c|c|}
\hline Variable Name & IBD $(\mathrm{N}=16550)$ & UC $(\mathrm{N}=7422)$ & $\mathrm{CD}(\mathrm{N}=9069)$ & Both $(\mathrm{N}=59)$ \\
\hline Age (Years) & $57.4 \pm 17.9$ & $59.7 \pm 18.3$ & $55.6 \pm 17.3$ & $53.2 \pm 17.3$ \\
\hline \multicolumn{5}{|c|}{ Race* $^{*}$} \\
\hline White & 80.5 & 80 & 80.9 & 78.6 \\
\hline Black & 11.2 & 9.8 & 12.3 & 14.3 \\
\hline Hispanic & 5 & 6.3 & 3.9 & 7.1 \\
\hline Asian/Pacific Islander & 0.7 & 1.1 & 0.4 & 0 \\
\hline Native American & 0.4 & 0.4 & 0.4 & 0 \\
\hline Other & 2.2 & 2.4 & 2.1 & 0 \\
\hline \multicolumn{5}{|c|}{ Gender } \\
\hline Male & 46.1 & 49.7 & 43.1 & 45.8 \\
\hline Female & 53.9 & 50.3 & 56.9 & 54.2 \\
\hline \multicolumn{5}{|c|}{ Admission Type ( $^{\$}$} \\
\hline Elective & 13.3 & 13.8 & 12.8 & 16.9 \\
\hline Non-elective & 86.7 & 86.2 & 87.2 & 83.1 \\
\hline \multicolumn{5}{|c|}{ Charlson’s/Deyo Comorbidity Index ${ }^{@}$} \\
\hline 0 & 49 & 48.9 & 49 & 57.6 \\
\hline 1 & 22.9 & 22.8 & 22.9 & 18.6 \\
\hline 2 & 12.3 & 11.8 & 12.7 & 11.9 \\
\hline$>3$ & 15.8 & 16.5 & 15.4 & 11.9 \\
\hline \multicolumn{5}{|c|}{ Primary Payer } \\
\hline Medicare & 47 & 47.3 & 46.9 & 25.4 \\
\hline Medicaid & 8.8 & 7.1 & 10.2 & 18.6 \\
\hline $\begin{array}{l}\text { Private Insurance (Including HMOs and } \\
\text { PPOs) }\end{array}$ & 37.5 & 39.1 & 36.1 & 42.4 \\
\hline Self-Pay & 3.1 & 2.9 & 3.2 & 8.5 \\
\hline Other & 3.6 & 3.6 & 3.6 & 5.1 \\
\hline \multicolumn{5}{|c|}{ Median Household Income for Patient's ZIP $\operatorname{code}^{\varphi}$} \\
\hline 0-25 Percentile & 22.5 & 20.3 & 24.3 & 17.5 \\
\hline 26-50 Percentile & 24.2 & 23.6 & 24.8 & 21 \\
\hline 51-75 Percentile & 25.7 & 25.9 & 25.6 & 31.6 \\
\hline 76-100 Percentile & 27.6 & 30.2 & 25.3 & 29.8 \\
\hline \multicolumn{5}{|c|}{ Teaching Status of Hospital } \\
\hline Rural & 7.8 & 6.8 & 8.6 & 5.1 \\
\hline Urban (Non-teaching) & 37.2 & 37.5 & 37 & 28.8 \\
\hline Urban (Teaching) & 55 & 55.6 & 54.5 & 66.1 \\
\hline \multicolumn{5}{|c|}{ Hospital Region } \\
\hline Northeast & 20.9 & 22.5 & 19.6 & 22 \\
\hline Midwest & 25 & 24 & 25.9 & 15.2 \\
\hline South & 35.7 & 32.7 & 38.2 & 32.2 \\
\hline West & 18.4 & 20.8 & 16.3 & 30.5 \\
\hline \multicolumn{5}{|c|}{ Disposition of Patient } \\
\hline Routine & 52.1 & 50.5 & 53.3 & 52.5 \\
\hline Transfer to Short-term Hospital & 3.3 & 3.4 & 3.2 & 3.4 \\
\hline Transfer to Other ${ }^{\$}$ & 19.7 & 22.3 & 17.7 & 13.6 \\
\hline Home Health Care & 19.5 & 17.6 & 21 & 28.8 \\
\hline Against Medical Advice & 0.6 & 0.4 & 0.8 & 0 \\
\hline
\end{tabular}




\begin{tabular}{|c|c|c|c|c|}
\hline Died & 4.7 & 5.7 & 3.9 & 1.7 \\
\hline Other: & 0.1 & 0.1 & 0.1 & 0 \\
\hline
\end{tabular}

*-2313 missing patients. \$- 24 missing patients.

@- Charlson's/Deyo comorbidity index was calculated as per Deyo classification.

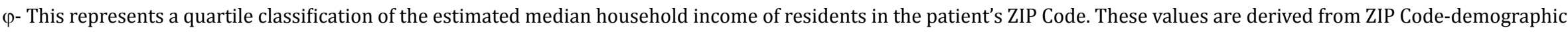

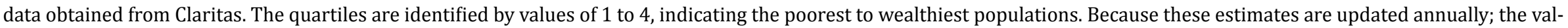
ue ranges vary by year. http://www.hcupus.ahrq.gov/db/vars/zipinc qrtl/nisnote.jsp.

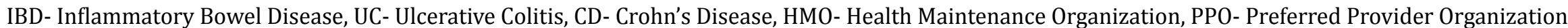

Table 2: Comorbidities of Patients Hospitalized with Venous Thromboembolism, Stratified by IBD, UC, CD and Both (in \%)

\begin{tabular}{|c|c|c|c|c|}
\hline Variable Name & IBD $(\mathrm{N}=16550)$ & $\mathrm{UC}(\mathrm{N}=7422)$ & $\mathrm{CD}(\mathrm{N}=9069)$ & Both $(\mathrm{N}=59)$ \\
\hline Diabetes Mellitus (uncomplicated) & 13.4 & 14.2 & 12.8 & 5.1 \\
\hline Diabetes Mellitus (with chronic complications) & 2.7 & 2.9 & 2.6 & 1.7 \\
\hline Hypertension & 39.7 & 41.8 & 38.1 & 27.1 \\
\hline Liver Disease & 4.5 & 5 & 4.1 & 1.7 \\
\hline Fluid and Electrolytes Disorder & 37.1 & 37.8 & 36.4 & 40.7 \\
\hline Other Neurological Disorder & 6.9 & 6.4 & 7.3 & 10.2 \\
\hline Obesity & 8.8 & 9.2 & 8.6 & 1.7 \\
\hline Peripheral Vascular Disease & 4.5 & 5.1 & 4 & 1.7 \\
\hline Valvular Disease & 3.7 & 3.8 & 3.7 & 1.7 \\
\hline Smoking & 16.2 & 12.6 & 19.2 & 11.9 \\
\hline Alcohol Abuse & 1.9 & 2.2 & 1.7 & 1.7 \\
\hline Coagulopathy & 9.8 & 10.5 & 9.2 & 13.5 \\
\hline Chronic Pulmonary Disease & 18.5 & 17.4 & 19.3 & 18.6 \\
\hline Congestive Heart Failure & 8.2 & 8.8 & 7.8 & 5.1 \\
\hline AIDS & 0.2 & 0.2 & 0.2 & 1.7 \\
\hline Deficiency Anemia & 31.6 & 30.1 & 32.9 & 37.3 \\
\hline Chronic Blood Loss Anemia & 5.1 & 6.5 & 3.9 & 6.8 \\
\hline Drug Abuse & 3 & 1.7 & 4 & 3.4 \\
\hline Renal Failure & 10.2 & 9.4 & 10.8 & 8.5 \\
\hline Peptic Ulcer Disease Excluding Bleeding & 0.1 & 0.1 & 0.1 & 0 \\
\hline Weight Loss & 18.3 & 18 & 18.5 & 16.9 \\
\hline
\end{tabular}

IBD- Inflammatory Bowel Disease, UC- Ulcerative Colitis, CD- Crohn's Disease, AIDS- Acquired Immuno Deficiency Syndrome

Table 3: Disease Presentation of Patients Hospitalized with Venous Thromboembolism -Stratified by IBD, UC, CD and Both. (in \%)

\begin{tabular}{|c|c|c|c|c|}
\hline Variable Name & IBD $(\mathrm{N}=16550)$ & UC $(\mathrm{N}=7422)$ & CD $(\mathrm{N}=9069)$ & Both $(\mathrm{N}=59)$ \\
\hline $\begin{array}{l}\text { Active Fistulizing disease or intra-abdominal } \\
\text { abscess }\end{array}$ & 7.1 & 4.4 & 9.3 & 10.2 \\
\hline Active Stricturing Disease & 2.6 & 1.3 & 3.7 & 1.7 \\
\hline Bowel Obstruction & 10.6 & 8 & 12.6 & 15.2 \\
\hline Perianal Abscess & 0.6 & 0.3 & 0.9 & 1.7 \\
\hline Unspecified Lower GI Bleeding & 5.2 & 6.1 & 4.4 & 10.2 \\
\hline Hypovolemia & 9.7 & 9.9 & 9.6 & 6.8 \\
\hline Malnutrition & 10.8 & 10.4 & 11.1 & 11.9 \\
\hline
\end{tabular}

IBD- Inflammatory Bowel Disease, UC- Ulcerative Colitis, CD- Crohn's Disease

Overall hospitalizations for IBD complicated by VTE are rising from 2005 to 2014 as shown in figure 2. Overall, in-hospital mortality was $4.7 \%$ in IBD patients complicated by VTE. This in-hospital mortality was higher in UC patients (5.6\%) compared to CD patients (3.9). In-hospital mortality in IBD patients was $0.18 \%$ in 2005 , which increased to $0.52 \%$ in 2014 (P for trend $=<0.0001$ ] [Figure 3]. In-Hospital mortality in UC patients was $0.2 \%$ in 2005 which increased to $0.56 \%$ in 2014 ( $\mathrm{P}$ for trend $=<0.0001$ ). In-Hospital mortality in CD patients was $0.16 \%$ in 2005 which increased to $0.49 \%$ in 2014 ( $\mathrm{P}$ for trend= $<0.0001$ ). Average length of stay was $10.7 \pm 13$ days in this patient population. Average length of stay in IBD patients was 9.6 days in 2005 which increased to 10.9 days in 2014 (P for trend $=<0.0001$ ). Trends of In-hospital Mortality in Patients Hospitalized with Venous Thromboembolism in IBD have been described in Table 4. IBD trends in patients who required surgery before and after 2008 is described in Figure 4. Reduction in VTE is noted after 2008 in IBD, UC and CD [Figure 4]. 


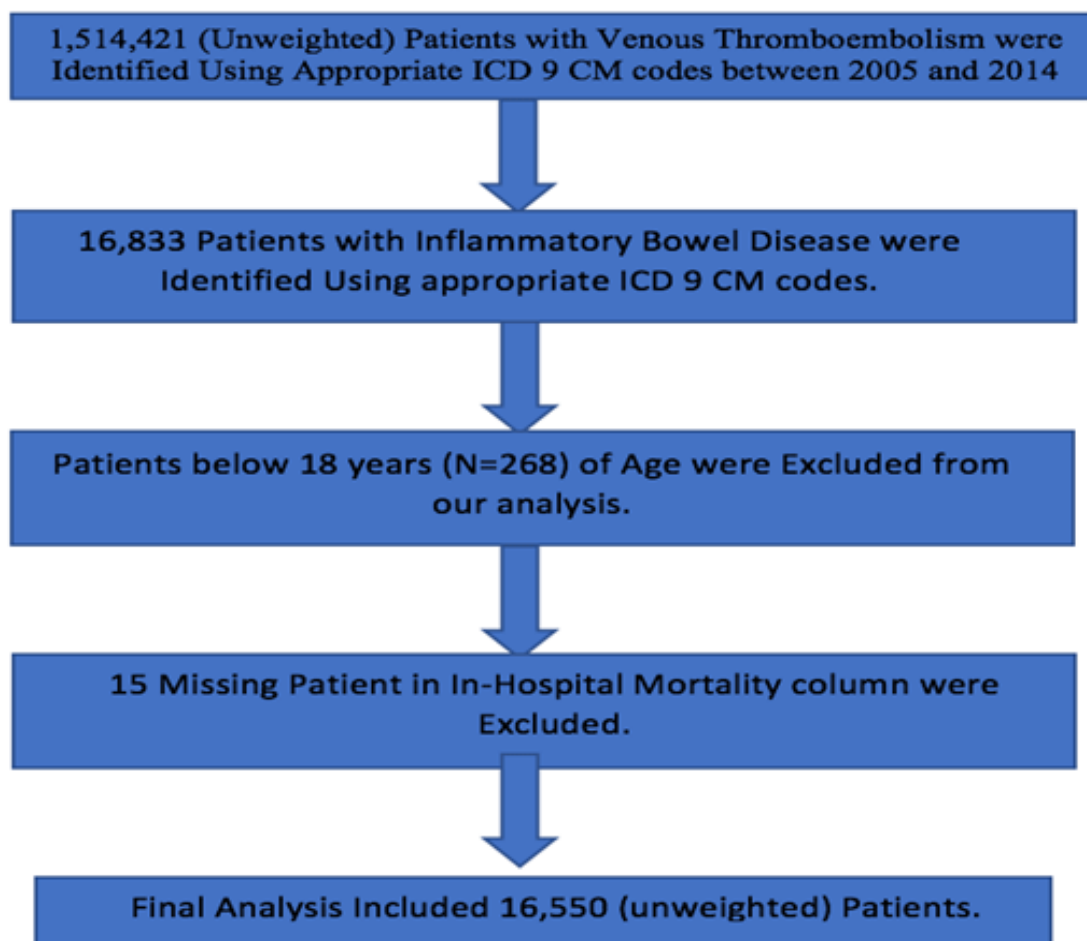

Figure 1: Patient Selection Chart

\section{Hospitalization Rate in US}

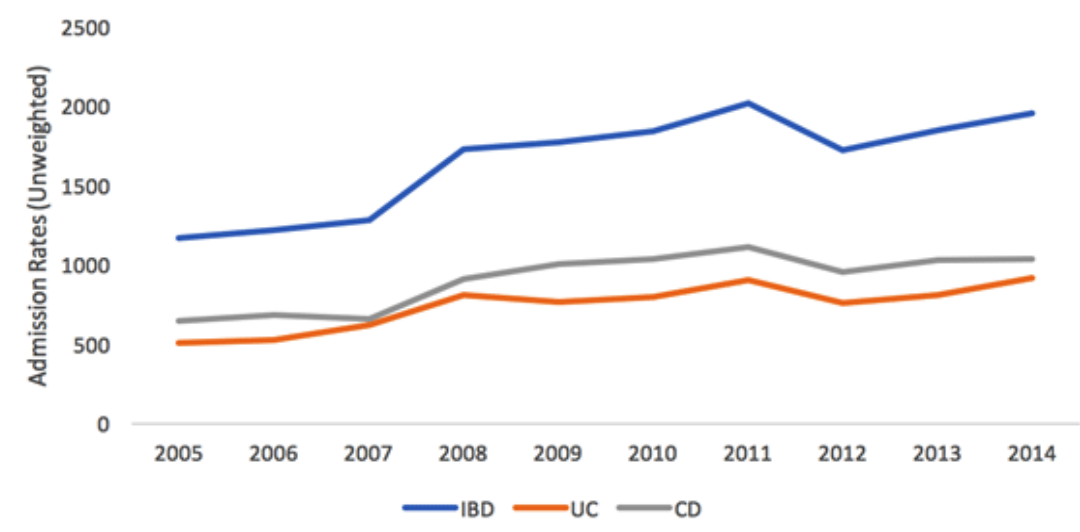

Figure 2: Trends in Hospitalization with Venous Thromboembolism Stratified by IBD, UC, CD

UC and CD cases were only available in 2014. Hence, we did not make a trend for that category.

IBD- Inflammatory Bowel Disease, UC- Ulcerative Colitis, CD- Crohn's Disease

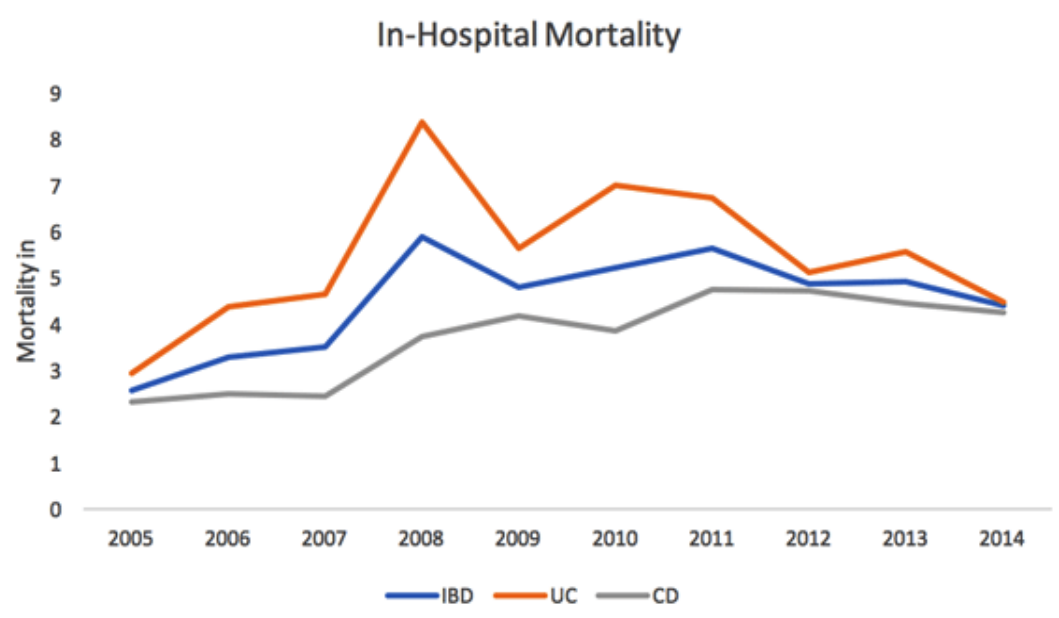

Figure 3: Trends in In-Hospital Mortality with Venous Thromboembolism - Stratified by IBD, UC, CD.

UC and CD cases were only available in 2014. Hence, we did not make a trend for that category.

IBD- Inflammatory Bowel Disease, UC- Ulcerative Colitis, CD- Crohn's Disease

\section{VTE in IBD who Required Surgery}

25

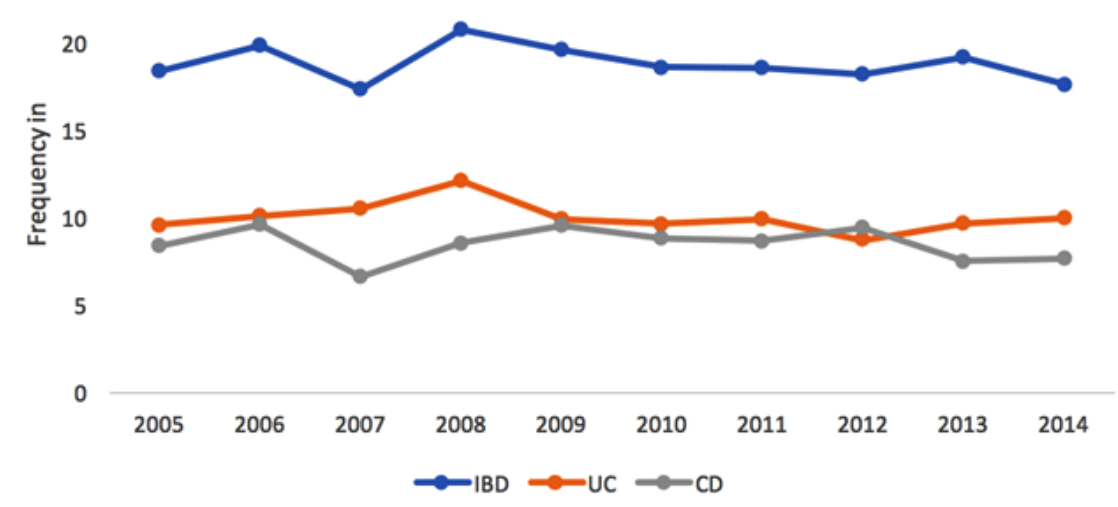

Figure 4: Trends with Venous Thromboembolism in Inflammatory Bowel Disease Patients Who Required Surgery: Stratified by IBD, UC, CD

UC and CD cases were only available in 2014. Hence, we did not make a trend for that category. Trending down after surgeon general's call to action in 2008 Abbreviations: IBD- Inflammatory Bowel Disease, UC- Ulcerative Colitis, CDCrohn's Disease

Surgeries Included for This Chart: Incision, Excision and Anastomosis of Intestine, Resection of Large Intestine, Rectal and perirectal Surgery, Other Surgery of small intestine, Other Surgery of large intestine, other surgery of intestine, unspecified site.

Table 4: Frequency and Trends of In-hospital Mortality in Patients Hospitalized with Venous Thromboembolism in IBD - Stratified by Years (in \%)

\begin{tabular}{|c|c|c|c|c|c|c|c|c|c|c|c|c|}
\hline Variable Name & 2005 & 2006 & 2007 & 2008 & 2009 & 2010 & 2011 & 2012 & 2013 & 2014 & Overall & $\begin{array}{l}\text { P value } \\
\text { (Trend) }\end{array}$ \\
\hline Unweighted Population & 1167 & 1217 & 1282 & 1729 & 1771 & 1841 & 2021 & 1721 & 1846 & 1955 & 16550 & $\mathrm{~N} / \mathrm{A}$ \\
\hline In-Hospital Mortality (Overall) & 2.57 & 3.29 & 3.51 & 5.9 & 4.8 & 5.21 & 5.64 & 4.88 & 4.93 & 4.4 & 4.67 & 0.005 \\
\hline \multicolumn{13}{|c|}{ Gender } \\
\hline Male & 1.28 & 1.48 & 1.4 & 2.7 & 2.5 & 2.3 & 2.6 & 2.3 & 2.3 & 1.9 & 2.2 & 0.1288 \\
\hline Female & 1.28 & 1.8 & 2.1 & 3.2 & 2.3 & 2.9 & 3 & 2.5 & 2.6 & 2.4 & 2.5 & 0.0157 \\
\hline \multicolumn{13}{|c|}{ Age } \\
\hline$<35$ & 0.1 & 0 & 0.1 & 0.2 & 0.2 & 0.3 & 0.2 & 0.3 & 0.1 & 0.1 & 0.2 & 0.4093 \\
\hline $35-50$ & 0.5 & 0.2 & 0.5 & 0.6 & 0.7 & 0.3 & 0.5 & 0.2 & 0.3 & 0.6 & 0.5 & 0.9366 \\
\hline $50-65$ & 0.5 & 0.6 & 0.8 & 1.3 & 0.9 & 1.7 & 1 & 1.5 & 0.2 & 0.9 & 1.1 & 0.072 \\
\hline $65-80$ & 0.5 & 1.7 & 0.8 & 2.8 & 1.9 & 1.5 & 2.6 & 2 & 2.2 & 1.8 & 1.9 & 0.0655 \\
\hline$>80$ & 0.9 & 0.6 & 1.2 & 0.9 & 1 & 1.4 & 1.3 & 0.9 & 1 & 0.9 & 1 & 0.5842 \\
\hline
\end{tabular}




\begin{tabular}{|c|c|c|c|c|c|c|c|c|c|c|c|c|}
\hline \multicolumn{13}{|c|}{ Race } \\
\hline White & 1.7 & 2.5 & 2.4 & 4.4 & 3.2 & 3.7 & 3.8 & 3.6 & 3.6 & 3.4 & 3.3 & 0.2623 \\
\hline Black & 0 & 0.3 & 0.3 & 0.3 & 0.6 & 0.6 & 0.8 & 0.5 & 0.3 & 0.2 & 0.4 & 0.7434 \\
\hline Other & 0.2 & 0.2 & 0.2 & 0.3 & 0.3 & 0.4 & 0.7 & 0.5 & 0.6 & 0.6 & 0.4 & 0.1144 \\
\hline \multicolumn{13}{|c|}{ Primary Payer } \\
\hline Medicare/Medicaid & 1.5 & 2.5 & 1.8 & 4 & 3.5 & 3.5 & 4.8 & 3.5 & 3.5 & 3.5 & 3.4 & 0.0127 \\
\hline $\begin{array}{l}\text { Private Insurance (Including } \\
\text { HMOs and PPOs) }\end{array}$ & 0.8 & 0.5 & 1.5 & 1.6 & 0.9 & 1.5 & 0.7 & 1 & 0.9 & 0.7 & 1 & 0.7299 \\
\hline Self-Pay/Other & 0.2 & 0.2 & 0.1 & 0.2 & 0.3 & 0.3 & 0.1 & 0.3 & 0.6 & 0.2 & 2.5 & 0.1169 \\
\hline \multicolumn{13}{|c|}{ Teaching Status of Hospital: } \\
\hline Rural & 0.3 & 0.1 & 0.2 & 0.5 & 0.3 & 0.4 & 0.2 & 0.3 & 0.4 & 0.2 & 0.3 & 0.1555 \\
\hline Urban (Non-teaching) & 1.1 & 1.1 & 1.3 & 2.1 & 1.8 & 2.3 & 2.3 & 1.3 & 1.5 & 0.8 & 1.6 & 0.0906 \\
\hline Urban (Teaching) & 1.2 & 2.1 & 1.9 & 3.3 & 2.4 & 2.5 & 2.9 & 3.3 & 3 & 3.3 & 2.7 & 0.108 \\
\hline \multicolumn{13}{|c|}{ Charlson's/Deyo Comorbidity Index } \\
\hline 0 & 0.8 & 0.9 & 1.1 & 1.7 & 1.5 & 1.8 & 1.5 & 1.7 & 1.4 & 1.3 & 1.4 & 0.0106 \\
\hline 1 & 0.8 & 1 & 0.8 & 1.3 & 1.2 & 1.1 & 1.4 & 1.2 & 1.2 & 1.2 & 1.1 & 0.107 \\
\hline 2 & 0.2 & 0.5 & 0.4 & 0.9 & 0.9 & 0.8 & 0.9 & 0.6 & 0.5 & 0.4 & 0.6 & 0.6032 \\
\hline$>3$ & 0.8 & 0.9 & 1.2 & 2 & 1.2 & 1.5 & 1.8 & 1.3 & 1.8 & 1.5 & 1.4 & 0.4 \\
\hline
\end{tabular}

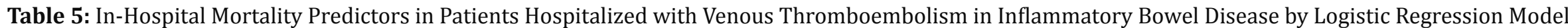

\begin{tabular}{|c|c|c|}
\hline Variable Name & Odds Ratio with $95 \%$ Confidence Interval & P value (Wald's) \\
\hline \multicolumn{3}{|c|}{ Age Groups } \\
\hline$<35$ & \multicolumn{2}{|c|}{ Referent } \\
\hline $35-50$ & $1.973(1.253-3.106)$ & 0.0033 \\
\hline $50-65$ & $3.220(2.112-4.911)$ & $<0.0001$ \\
\hline $65-80$ & $5.441(3.485-8.497)$ & $<0.0001$ \\
\hline$>80$ & $6.933(4.367-11.007)$ & $<0.0001$ \\
\hline Female vs Male & $0.956(0.823-1.109)$ & 0.5516 \\
\hline Elective vs Non-Elective & $0.810(0.637-1.030)$ & 0.0862 \\
\hline \multicolumn{3}{|c|}{ Primary Payer } \\
\hline Medicare & \multicolumn{2}{|c|}{ Referent } \\
\hline Medicaid & $1.088(0.758-1.560)$ & 0.6485 \\
\hline Private Including HMOs and PPOs & $0.819(0.650-1.032)$ & 0.0909 \\
\hline Self-Pay/No-Charge/other & $1.282(0.758-2.168)$ & 0.3551 \\
\hline \multicolumn{3}{|c|}{ Hospital Location } \\
\hline Rural & Referent & \\
\hline Urban Non-teaching & $1.157(0.847-1.581)$ & 0.3595 \\
\hline Urban Teaching & $1.429(1.056-1.934)$ & 0.0277 \\
\hline \multicolumn{3}{|c|}{ Charlson's/Deyo Comorbidity Index } \\
\hline 0 & \multicolumn{2}{|c|}{ Referent } \\
\hline 1 & $1.396(1.142-1.705)$ & 0.0011 \\
\hline 2 & $1.330(1.046-1.691)$ & 0.0201 \\
\hline$>3$ & $2.411(1.987-2.925)$ & $<0.0001$ \\
\hline
\end{tabular}

Multivariate predictor model for in-hospital mortality was generated to assess for predictors of in-hospital mortality in IBD patients complicated with VTE [Table 5]. After adjusting for various confounders, increased age was associated with a greater risk of in-hospital mortality. Other predictors included treatment in urban teaching hospital and higher Charlson's comorbidity index.

\section{Discussion}

Using nationwide population based hospital data from the United States; we have confirmed previous findings that VTE is associated with higher mortality and increased length of hospital stay in IBD patients. The occurrence of VTEs is an increasingly recognized source of extra intestinal morbidity and mortality among IBD patients, even after recent advancement for treatment of IBD and prevention of VTE. The most common comorbid conditions in our study were hypertension, fluid and 
electrolyte disorder, deficiency anemia, and chronic pulmonary disease. The data also showed that malnutrition, bowel obstruction, hypovolemia, active fistulizing disease and intraabdominal abscess were commonly present.

In our study, majority of the population with VTE in IBD had low CCI index which proposed that IBD could be an independent risk factor for VTE as suggested in previous studies. But CCI $>3$ is strongly associated with higher mortality which demands screening for high risk for VTE in IBD population. Interestingly, our data suggest that there is higher rate of VTE in IBD at urban teaching hospitals. Our finding is consistent with the previous study that showed 14\% - 15\% high likelihood of having VTE in urban hospitals compared to rural hospitals [14]. It may be due to having more debilitated patients in urban tertiary centers who are at the greater risk for VTE.

Among the comorbidities, the most common was hypertension. It is associated with endothelial injury, a part of Virchow's triad. Hypertension could be an independent risk factor for VTEs which may be mediated by the presence of inflammation. It is proven in recent study of newly diagnosed lung cancer patients [15]. Another significant comorbidity was fluid and electrolyte disorder which is common in IBD patients during flare. It could be due to inadequate oral intake. Dehydration is associated with hyper viscosity and ultimately increases risk of thrombosis. In recent study, dehydration is strongly associated with VTE after acute ischemic stroke $[16,17]$. It should be further studied in IBD patients for a risk of VTE. Iron deficiency and anemia of chronic disease is also a well-known complication of IBD. It is due to the combination of malabsorption and chronic inflammatory state. It might be associated with increased risk of VTE in IBD. It should be investigated when anemia is a proven risk factor for VTE and cerebral venous thrombosis [17].

The most clinically relevant finding is the increasing VTE-associated mortality in IBD patients from 2005 to 2014. Although, IBD patients are younger and have less comorbid conditions, our finding shows an increase in twofold mortality. This finding may be an indication that VTEs are more severe in IBD patients. The rapidly rising rate of VTE in the hospitalized UC and CD population may be due to corticosteroid use and merits further exploration [18]. IBD related surgeries are unlikely the cause of mortality because the rate of VTE in IBD who required surgery has been decreased since 2008 as shown in chart 6 . This may be due to efforts from office of surgeon general to prevent perioperative DVT and PE in 2008 [19]. With new biologic therapy thought to be associated with lower risk of VTE, it will need more studies [20]. Also, the rise in VTE rates may be attributable to advancement in diagnostic testing. Another possible explanation is that patients with UC and Crohn's colitis may be more likely to have hematochezia and subsequently less likely to receive VTE prophylaxis.

Interestingly, it appears that UC patients with VTE have higher mortality compared to CD patients with VTE. This greater mortality among UC patients was previously suggested in a population-based study which could be explain further by higher colonic involvement and inflammation being a predisposing factor for VTE [21]. However, we do not have endoscopic data to comment on the role of severity of colonic inflammation. Supplementary studies are warranted to characterize the mechanisms of these associations. Our secondary outcomes include increased complication rate which could be attributing to the increased length of stay.

The increase in mortality for IBD patients with VTE emphasizes the potential benefit of adequate VTE prophylaxis. Further studies need to assess whether screening strategies for VTE in IBD patients. Despite reasonable awareness of the increased risk of thrombosis in hospitalized IBD patients, many clinicians may not follow clinical practice guidelines and use pharmacologic VTE prophylaxis [22]. Studies are warranted to measure the rate of VTE prophylaxis among hospitalized IBD patients. Even recent study shows that prophylactic anticoagulation is safe in IBD despite the presence of rectal bleeding on admission [23]. Thus, unless in the presence of hemodynamically significant GI bleeding, unfractionated or low-molecular weight heparin should not be contraindicated in either the treatment or prophylaxis of venous thrombosis. VTE is an increasingly prevalent and preventable complication of IBD and efforts toward early detection and therapy are essential in improving outcomes for IBD inpatients.

The large sample size of the NIS data set is a population-based representation of all hospitalized IBD patients in the United States and reflects all types of hospital settings, insurance payers, and geographic regions. Thus, the selected study population enhances the generalizability.

\section{Limitations}

The NIS is publically accessible dataset and contains completely de-identified data. The use of such administrative data sets has its own limitation like the data are not specifically collected to answer a research question, restricted to in-patient stays only and does not track each patient longitudinally and they are also subject to misclassification. Administrative discharge codes are validated for the analysis of various diseases and their outcomes. The NIS considers each hospitalization as separate entry so it is not possible to separate index cases from readmissions. This could result in an overestimation of the number of admissions. However, we have the largest patient population and absence of selection bias associated with clinical trials. Also, white subjects (80.5\%) were predominant; female subjects were higher compared to male subjects and Regional difference was also noted that highest subjects were studied from South America region (35.7\%) compared to other hospital region. It suggests that distribution of patients was not equal in terms of gender, geographical location and race. Despite the limitations, inclusion of a huge number of patients from various demographic backgrounds, patients from all types of incomes, all types of insurances and from academic and nonacademic institutions makes the results highly generalizable as it represents a "realworld" population.

\section{Conclusion}

We demonstrated an increasing trend in the rate of VTE associated with IBD hospitalization among US adults. However, it is not possible to determine if this increased trend in VTE are associated with increased diagnosis and awareness or a true increase in the incidence. Importantly, since the surgeon general's call to action in 2008, reduction in inhospital mortality has been noted in IBD patients underwent surgery and occurrence of VTE. The result of this study re-emphasizes the need of continued research; improve prevention and therapeutic management 
of VTE in IBD patients. Future research should be directed towards prevention of VTE, better therapeutic management which can lead to reduction in in-hospital mortality and long term benefits.

\section{References}

1. Monsen U, Sorstad J, Hellers G and Johansson C. Extracolonic diagnoses in ulcerative colitis: an epidemiological study. Am J Gastroenterol. 1990;85(6):711-716.

2. Bargen J and Barker NW. Extensive arterial and venous thrombosis complicating chronic ulcerative colitis. Arch Intern Med. 1936;58(1):17-31. Doi:10.1001/ archinte.1936.00170110025002

3. Bernstein CN, Blanchard JF, Houston DS and Wajda A. The incidence of deep venous thrombosis and pulmonary embolism among patients with inflammatory bowel disease: a population-based cohort study. Thromb Haemost. 2001;85(3):430-434.

4. Grip 0, Svensson PJ and Lindgren S. Inflammatory bowel disease promotes venous thrombosis earlier in life. Scand J Gastroenterol. 2000;35(6):619-623.

5. Miehsler W, Reinisch W, Valic E, Osterode W, Tillinger W, Feichtenschlager T, Grisar J, et al.Is inflammatory bowel disease an independent and disease specific risk factor for thromboembolism? Gut. 2004;53(4):542-548. doi: 10.1136/gut.2003.025411

6. Danese S, Papa A, Saibeni S, Repici A, Malesci A and Vecchi M. Inflammation and coagulation in inflammatory bowel disease: The clot thickens. Am J Gastroenterol 2007;102(1):174-186.

7. Cushman M, Tsai AW, White RH, Heckbert SR, Rosamond WD, Enright P and Folsom AR. Deep vein thrombosis and pulmonary embolism in two cohorts: the longitudinal investigation of thromboembolism etiology. Am J Med. 2004;117(1):19-25.

8. Goldhaber SZ, Visani L and De Rosa M. Acute pulmonary embolism: clinical outcomes in the International Cooperative Pulmonary Embolism Registry (ICOPER). Lancet. 1999;353(9162):1386-1389.

9. Kearon C, Kahn SR, Agnelli G, Goldhaber S, Raskob GE, Comerota AJ and American College of Chest P. Antithrombotic therapy for venous thromboembolic disease: American College of Chest Physicians Evidence-Based Clinical Practice Guidelines (8th Edition). Chest. 2008;133(6):454S-545S. doi: 10.1378/chest.08-0658

10.Palareti G and Cosmi B. Bleeding with anticoagulation therapy - who is at risk, and how best to identify such patients. Thromb Haemost. 2009;102(2):268-278. doi: 10.1160/ TH08-11-0730

11.Rockville. Nationwide Inpatient Sample, Healthcare Cost and Utilization Project (HCUP). Quality AfHRa.
12.Elixhauser A, Steiner C, Harris DR and Coffey RM. Comorbidity measures for use with administrative data. Med Care. 1998;36(1):8-27.

13.Ananthakrishnan AN, McGinley EL and Binion DG. Does it matter where you are hospitalized for inflammatory bowel disease? A nationwide analysis of hospital volume. Am J Gastroenterol. 2008;103(11):2789-2798.

14.Tsai J, Grant AM, Beckman MG, Grosse SD, Yusuf HR and Richardson LC. Determinants of venous thromboembolism among hospitalizations of US adults: a multilevel analysis. PloS one. 2015;10(4):e0123842. doi: 10.1371/journal.pone.0123842

15.Zhang Y, Yang Y, Chen W, Liang L, Zhai Z, Guo L, Wang C and China Venous Thromboembolism VTESG. Hypertension associated with venous thromboembolism in patients with newly diagnosed lung cancer. Sci Rep. 2016;6:19603. doi: 10.1038/ srep19603

16. Kelly J, Hunt BJ, Lewis RR, Swaminathan R, Moody A, Seed PT and Rudd A. Dehydration and venous thromboembolism after acute stroke. QJM : monthly journal of the Association of Physicians. 2004;97(5):293-296.

17.Hung SH, Lin HC and Chung SD. Association between venous thromboembolism and iron-deficiency anemia: a population-based study. Blood coagulation \& fibrinolysis. 2015;26(4):368-372. doi: 10.1097/MBC.0000000000000249

18.Waljee AK, Wiitala WL, Govani S, Stidham R, Saini S, Hou J, Feagins LA, et al. Corticosteroid Use and Complications in a US Inflammatory Bowel Disease Cohort. PloS one. 2016;11(6):e0158017. doi: 10.1371/journal.pone.0158017

19.Rockville. The Surgeon General's Call to Action to Prevent Deep Vein Thrombosis and Pulmonary Embolism. Publications and Reports of the Surgeon General. 2008.

20.Higgins PD, Skup M, Mulani PM, Lin J and Chao J. Increased risk of venous thromboembolic events with corticosteroid vs biologic therapy for inflammatory bowel disease. Clin Gastroenterol Hepatol. 2015;13(2):316-321. doi: 10.1016/j.cgh.2014.07.017

21.Bernstein CN, Wajda A, Svenson LW, MacKenzie A, Koehoorn M, Jackson M, Fedorak R, Israel D and Blanchard JF. The epidemiology of inflammatory bowel disease in Canada: a population-based study. Am J Gastroenterol. 2006;101(7):1559-1568.

22.Tinsley A, Naymagon S, Trindade AJ, Sachar DB, Sands BE and Ullman TA. A survey of current practice of venous thromboembolism prophylaxis in hospitalized inflammatory bowel disease patients in the United States. J Clin Gastroenterol. 2013;47(1):e1-6. doi: 10.1097/MCG.0b013e31824c0dea

23.Ra G, Thanabalan R, Ratneswaran S and Nguyen GC. Predictors and safety of venous thromboembolism prophylaxis among hospitalized inflammatory bowel disease patients. J Crohns Colitis. 2013;7(10):479-485. doi: 10.1016/j.crohns.2013.03.002 